\title{
p53 is a regulator of macrophage differentiation
}

\author{
D Matas ${ }^{1}$, M Milyavsky ${ }^{1}$, I Shats ${ }^{1}$, L Nissim ${ }^{1}$, N Goldfinger $^{1}$ and \\ V Rotter ${ }^{*, 1}$ \\ ${ }^{1}$ Department of Molecular Cell Biology, The Weizmann Institute of Science, \\ Rehovot, Israel \\ * Correspondening Author: V Rotter, Department of Molecular Cell Biology, \\ Weizmann Institute of Science, Rehovot 76100, Israel. Tel: + 972-8-9344501/ \\ + 972-8-9466264; Fax: +972-08-9465265; \\ E-mail: varda.rotter@weizmann.ac.il
}

Received 25.5.03; revised 15.9.03; accepted 17.11.03; published online 9.1.04 Editted by D Altieri

\section{Abstract \\ While it is well accepted that p53 plays a role in apoptosis, less is known as to its involvement in cell differentiation. Here we show that wild-type p53 facilitates IL-6-dependent macrophage differentiation. Treatment of M1/2 cells expres- sing the temperature-sensitive p53 143 (Val to Ala) mutant, at the wild-type conformation, facilitated the appearance of mature macrophages that exhibited phagocytic activity. Enhancement of differentiation by the p53 143 (Val to Ala) in the wild-type conformation was coupled with the inhibition of apoptosis induction by this protein. In agreement with previous studies, we found that p53 levels were reduced during p53-dependent macrophage differentiation. This occurred when p53 levels before IL-6 stimuli were high. Interestingly, the p53 143 (Val to Ala) protein, at the mutant conformation, enhanced macrophage differentiation, as did the wild-type conformation, whereas the p53 273 (Arg to His) core mutant exerted an inhibitory effect on this pathway. The transcription-deficient p53 molecules, p53 (22-23) and p53 22,23,143, could not induce p53-dependent differentiation. Moreover, the p53 (22-23) protein inhibited the p53- independent differentiation pathway. Interestingly, the p53 (22-23) protein not only blocked IL-6-mediated differentiation, but also induced significant apoptotic cell death, upon IL-6 stimulation. Taken together, our data show that wild-type p53 enhances macrophage differentiation, while various p53 mutant types exert different effects on this differentiation pathway. \\ Cell Death and Differentiation (2004) 11, 458-467. doi:10.1038/ sj.cdd.4401379 \\ Published online 9 January 2004}

Keywords: p53 tumor-suppressor gene; mutant p53; macrophage cell differentiation; telomerase

\footnotetext{
Abbreviations: AO, Acridine orange; DMEM, Dulbecco's modified Eagle's medium; HBSS, Hank's balanced salt solution; HRP, Horseradish peroxidase; IL-6, interleukin-6; TRAP, telomeric repeat amplification protocol
}

\section{Introduction}

The differentiation of human hematopoietic cells is a multistep process that is essential for the generation of mature blood cells. ${ }^{1}$ Hematopoietic myeloid precursor cells differentiate to form mature granulocytes and macrophages. After exerting their functions, the differentiated cells die via apoptosis. ${ }^{2}$ The molecular events that underlie the production of mature myeloid cells and the preceding apoptotic process are not well understood. However, studies of myeloid differentiation suggest that the modulation of this process is mainly at the level of mRNA transcription. ${ }^{3}$

The p53 tumor-suppressor gene plays a central role in the regulation of cell fate. p53 is involved in a variety of cell cyclerelated processes including the activation of cell cycle checkpoints, ${ }^{4,5}$ apoptosis, ${ }^{6-8}$ cellular senescence, ${ }^{9}$ DNA repair $^{10-12}$ and cell differentiation. ${ }^{13}$ The involvement of p53 in cellular differentiation and embryonic development is less characterized than its role in growth arrest and apoptosis. However, p53 has been implicated in the regulation of these processes by studies of both in vitro and in vivo models. Although it was initially reported that p53-null mice develop normally, ${ }^{14}$ more detailed analysis pointed to the conclusion that these mice suffer at certain frequencies from developmental abnormalities. ${ }^{15,16}$ In addition to developmental defects, p53 deficiency affects the differentiation of various cell types (reviewed in Almog and Rotter ${ }^{13}$ and in Choi and Donehower ${ }^{17}$ ). Similarly, introduction or overexpression of p53 in several tumor cell lines induces limited differentiation. Involvement of p53 in differentiation was also demonstrated in hematopoietic cells. It appears that p53 is involved in the differentiation of both the lymphoid and myeloid lineages (reviewed in Almog and Rotter ${ }^{13}$ ). Studies in our laboratory have demonstrated a role for p53 in B-cell differentiation. ${ }^{18-20}$ Expression of p53 in HL-60 promyelocytic cell line induced differentiation along the granulocytic ${ }^{21}$ or the monocytic pathways. $^{22,65}$ p53 was also implicated in erythroid differentiation. ${ }^{23-26}$

The mechanism that underlies the involvement of p53 in differentiation is poorly understood. Several reports showed that endogenous p53 levels and activities are regulated upon differentiation. p53 expression was shown to increase during the maturation of human hematopoietic cells. ${ }^{27}$ On the contrary, a decrease in p53 protein levels was observed upon the differentiation of epidermal keratinocytes, ${ }^{28}$ embryonal carcinoma cells ${ }^{29}$ and embryonic stem cell lines. ${ }^{30}$ Interestingly, the reduction in p53 expression in epidermal keratinocytes $^{28}$ and embryonal carcinoma cells ${ }^{29}$ was accompanied by an increase in the transcriptional activity of the p53 protein. It appears that the involvement of p53 in cell differentiation is mediated through its transcriptional activity. The identification of differentiation-induced genes that are transactivated by $\mathrm{p} 53$, in reporter assays, further supports this notion. ${ }^{31-33}$

Alterations of the p53 gene are the most common genetic defect in human cancer. ${ }^{34}$ Most prevalent are missense mutations, which give rise to full-length, yet mutant versions of the protein, expressed at very high levels. Mutant p53 was 
shown to repress wild-type p53-mediated differentiation in cells expressing both alleles. ${ }^{20,25,31}$ Several studies have shown that, in cells devoid of wild-type p53, the expression of mutant p53 inhibits differentiation. ${ }^{19}$ Moreover, in clinical studies of cancer patients, mutations in p53 frequently correlate with undifferentiated phenotypes of cancers. ${ }^{35,36}$

The M1 murine myeloblastic cell line, which is p53-null, can be induced by interleukin-6 (IL-6) to undergo terminal macrophage differentiation. ${ }^{37}$ We found that a derivative of this line, the $M 1 / 2$ cell line, ${ }^{38}$ retains the capacity to differentiate in response to IL-6. We utilized the M1/2 p53null cell line and $\mathrm{M} 1 / 2$ stable clones expressing various human p53 forms to investigate the role of p53 in IL-6-mediated myeloid differentiation. Using $\mathrm{M} 1 / 2$-derived clones expressing the p53 143 (Val to Ala) temperature-sensitive mutant, we demonstrate that p53 enhances myeloid differentiation. Surprisingly, this effect was observed both at the wild-type and at the mutant conformation. At $32^{\circ} \mathrm{C}$, the permissive temperature for wild-type p53 expression, IL-6 treatment resulted both in the inhibition of p53-mediated apoptosis and in the facilitation of p53-mediated differentiation. On the contrary, we observed that the p53 273 (Arg to His) bona fide core mutant inhibits myeloid differentiation to some extent. We show that p53-dependent myeloid differentiation requires the transcriptional activity of p53. Moreover, transcriptionally inactive p53, p53 (22-23), interferes with p53-independent myeloid differentiation of $\mathrm{M} 1 / 2$ cells and induces apoptosis upon IL-6 treatment. Interestingly, p53-dependent differentiation is accompanied by decrease in 053 protein expression, when the expression before IL-6 stimuli is high. Our results show that, while wild-type p53 facilitates macrophage differentiation, various p53 mutant forms affect this pathway differently.

\section{Results}

In this study, we have investigated the involvement of p53 in myeloid differentiation. For that purpose, we utilized the $M 1 / 2$ p53 nonproducer murine myeloid cell line and M1/2-derived clones expressing various human p53 mutants. ${ }^{39}$ First, we examined whether the $M 1 / 2$ cells retained the ability to differentiate in response to IL-6, as observed with the parental M1 murine myeloblastic cell line. ${ }^{37}$ We found that stimulation of M1/2 cells with IL-6 induced the typical blast morphology of macrophage differentiation, as assessed by Giemsa staining (see Figure $2 \mathrm{a}$, vector at $37^{\circ} \mathrm{C}$ ). Thus, the $\mathrm{M} 1 / 2$ cell line can serve as a model system for myeloid differentiation.

\section{Effect of IL-6 on p53 143 (Val to Ala) producer, M1/2 cells}

To study the effect of wild-type p53 on myeloid differentiation, we utilized M1/2 clones expressing the p53 143 (Val to Ala) protein, which is known as a temperature-sensitive (ts) mutant. ${ }^{40}$ Temperature shift of these clones to $32^{\circ} \mathrm{C}$ induces apoptotic cell death, due to wild-type p53 expression. Previously, it was demonstrated that apoptosis induced in M1 cells by the expression of the temperature-sensitive murine mutant p53 135 (Ala to $\mathrm{Val}$ ) at $32^{\circ} \mathrm{C}$ is blocked by

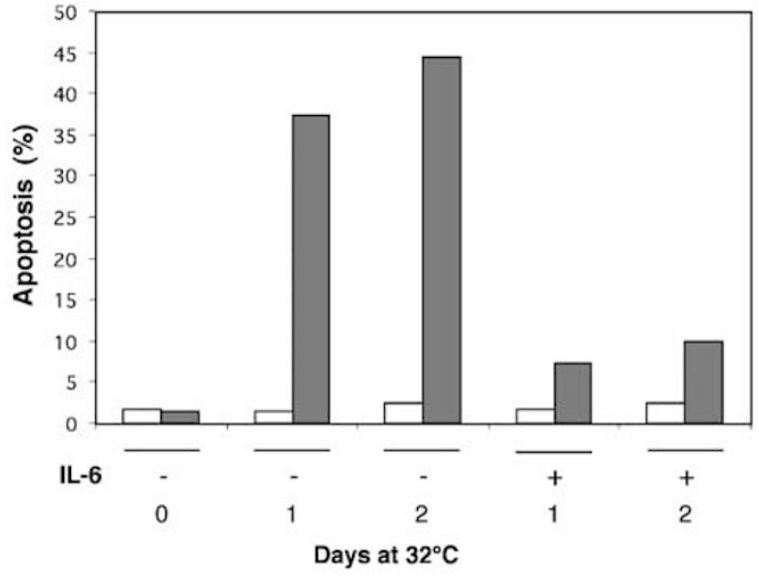

Figure 1 IL-6 inhibits apoptosis induction by the p53 143 (Val to Ala) protein in its wild-type conformation. Apoptosis percentage of M1/2-derived clones expressing the p53 143 (Val to Ala) protein, p53-143-106 (dark bars), or the empty vector (empty bars) upon shift to $32^{\circ} \mathrm{C}$ for the time points indicated, with or without pretreatment with IL-6. Apoptosis was quantitated by the FACS-based AO DNA denaturability assay

pretreatment with IL-6. ${ }^{41}$ We examined whether the p53 143 (Val to Ala) protein expressed in $M 1 / 2$ cells behaves in a similar manner. For that we analyzed the effect of treatment with IL-6 on the apoptotic percentage of p53 143 (Val to Ala) expressing $\mathrm{M} 1 / 2$ cells, at $32^{\circ} \mathrm{C}$. Empty vector-expressing cells were included as a control. Apoptosis induction was analyzed by the FACS-based acridine orange (AO) DNA denaturability assay. ${ }^{42}$ In agreement with previous studies, we found that in the $M 1 / 2$ derivative treatment with IL- 6 before the shift to $32^{\circ} \mathrm{C}$ significantly reduces the apoptosis percentage of p53 143 (Val to Ala)-expressing cells (Figure 1). Thus, IL-6 inhibits the apoptotic process induced by wild-type p53. These results enabled the analysis of wild-type p53 effect on IL-6-induced myeloid differentiation, utilizing the $M 1 / 2$ clones expressing the temperature-sensitive p53.

It was of interest to examine whether the p53 143 (Val to Ala)- expressing cells underwent cell differentiation, instead of apoptosis. To that end, M1/2 cells expressing the p53 143 (Val to Ala) protein were treated with IL-6, shifted to $32^{\circ} \mathrm{C}$, the permissive temperature for the wild-type p53 conformation, and 3 days later the differentiation status was examined by Giemsa staining (Figure 2a). Similar experiments were carried out at $37^{\circ} \mathrm{C}$, to determine the effect of the p53 143 (Val to Ala) in the mutant conformation on the differentiation process. We found that nontreated $\mathrm{M} 1 / 2$ cells expressing either the empty vector or the p53 143 (Val to Ala) protein exhibited 2-4\% differentiated cells. Interestingly, upon IL-6 treatment, both at $32^{\circ} \mathrm{C}$ and at $37^{\circ} \mathrm{C}$, the p53 143 (Val to Ala)-expressing clone exhibited significantly more differentiating cells than the empty vector control clone. At $32^{\circ} \mathrm{C}, 24 \%$ of the control cells exhibited macrophage-like morphology, while $49 \%$ differentiated cells were found in the p53 143 (Val to Ala)-expressing cells. Likewise, upon IL- 6 treatment at $37^{\circ} \mathrm{C}, 40 \%$ of the control cells underwent differentiation, while in the p53 143 (Val to Ala)-expressing clone the differentiated population reached $90 \%$. Figure 2 a depicts a representative picture of the Giemsa results at $37^{\circ} \mathrm{C}$ 
a
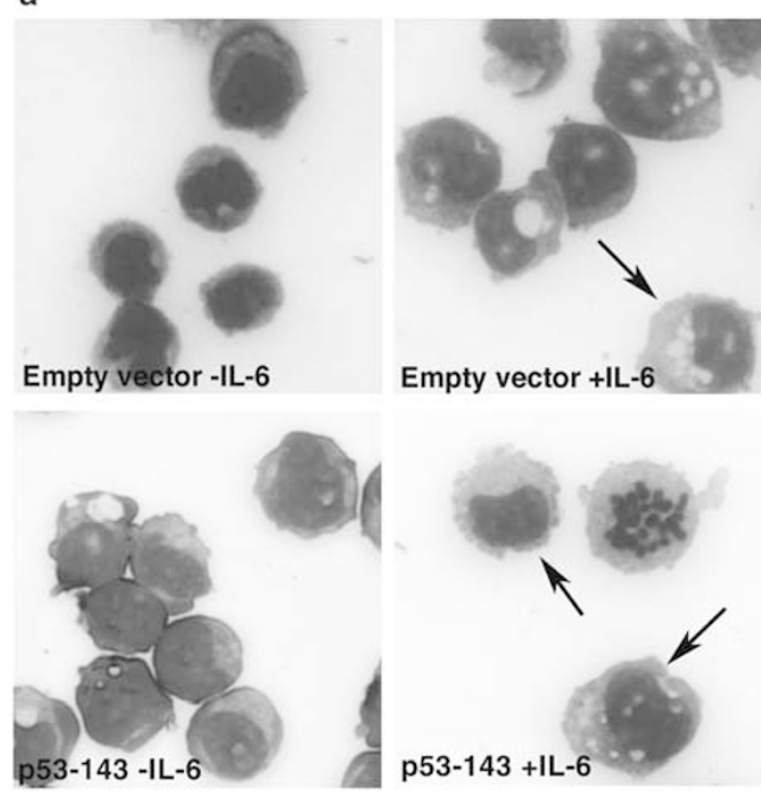

b

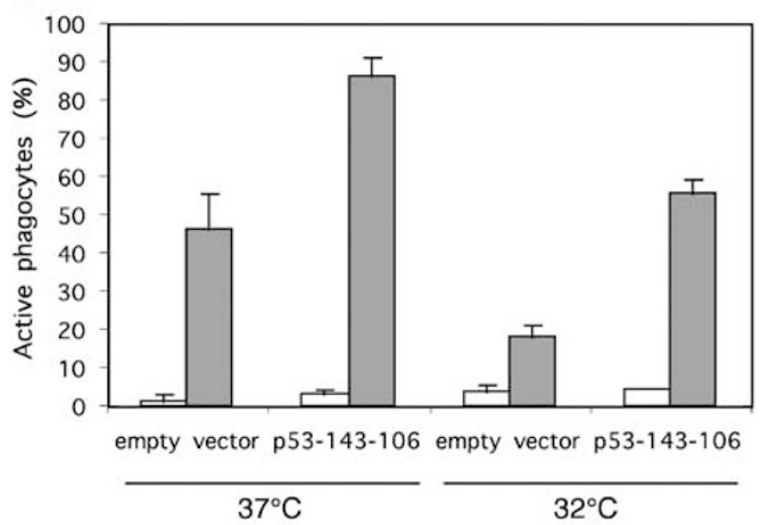

Figure 2 Enhancement of myeloid differentiation by the p53 143 (Val to Ala) protein. A comparison of the differentiation pattern in $\mathrm{M} 1 / 2$-derived clones expressing the p53 143 (Val to Ala) protein or the empty vector after IL-6 treatment and incubation at $32^{\circ} \mathrm{C}$ or at $37^{\circ} \mathrm{C}$ for 3 days. Cytocentrifuge preparations of M1/2-derived clones were stained with Giemsa to evaluate morphological features at $37^{\circ} \mathrm{C}$. Differentiation to macrophages is characterized by enlarged cell size, vacuoles in the cytoplasm and a smaller ratio of the nucleus/cytoplasm. Arrows indicate examples of cells that exhibit macrophagelike morphology. (a). Quantitation of the capacity of M1/2 clones to phagocytose Alexa-conjugated E.coli bioparticles following IL-6 stimulation and incubation at $37^{\circ} \mathrm{C}$ or shift to $32^{\circ} \mathrm{C}$. The empty bars represent nontreated cells and the dark bars represent cells treated with IL-6 (b)

Next, it was of interest to determine how functional activities typical to mature macrophages are modulated in the presence and absence of the p53 143 ( Val to Ala) protein. The phagocytic activity of the cells, a hallmark of macrophage differentiation, was measured. The ability of the M1/2 cells to phagocytose opsonized Alexa-labeled E. coli bioparticles was employed to measure their phagocytic capacity. In agreement with the Giemsa stains, we observed at $32^{\circ} \mathrm{C}$ that the p53 143 (Val to Ala) in the wild-type conformation dramatically enhanced macrophage differentiation of $M 1 / 2$ cells (see Figure 2b). The p53 143 (Val to Ala)-expressing clone

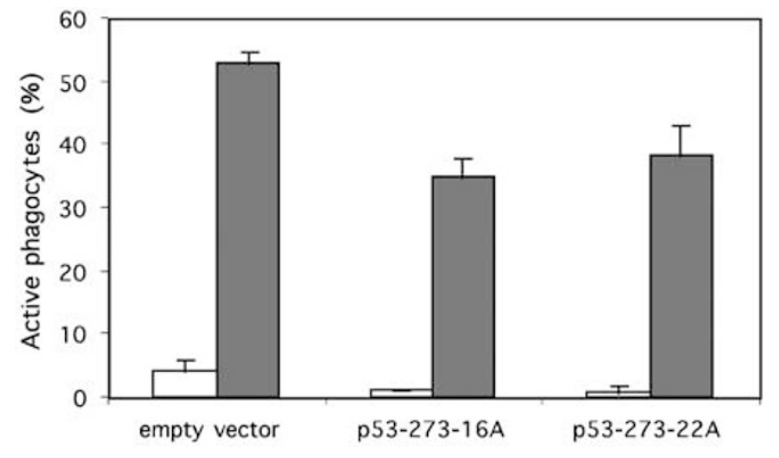

Figure 3 The p53 273 (Arg to His) protein interferes with myeloid differentiation. Comparison of the phagocytic activity of M1/2 cells expressing either the empty vector or the p53 273 (Arg to His) mutant, after IL-6 stimulation for 3 days (dark bars) or without IL-6 treatment (empty bars). Two clones expressing the p53 273 (Arg to His) mutant were utilized: the p53-273-16A and the p53-273-22A

exhibited about three-fold of differentiating cells, compared to the empty vector control. Again, at $37^{\circ} \mathrm{C}$, mutant p53 conformation temperature, the p53 143 (Val to Ala) facilitated the differentiation process significantly. Thus, we concluded that the p53 143 (Val to Ala) protein exerted a promoting effect on myeloid differentiation. This effect was observed both at $32^{\circ} \mathrm{C}$ and at $37^{\circ} \mathrm{C}$.

\section{Effect of p53 273 (Arg to His) expression on myeloid differentiation}

It is worth mentioning that the p53 143 (Val to Ala) temperature-sensitive mutant induces apoptosis, a wild-type p53 feature, at $32^{\circ} \mathrm{C}$, and is not involved in apoptosis at $37^{\circ} \mathrm{C}$. The observation that, in the differentiation process, no differences were observed between the two temperatures raises the possibility that temperature-induced conformational changes in p53 are less significant in the latter pathway. Therefore, to assess the effect of mutant p53 on differentiation, we next focused on the bona fide p53 mutant 273 (Arg to His), one of the most frequent mutations in p53 observed in human cancer cells. Cells expressing either the empty vector or the p53273 (Arg to His) mutant were stimulated with IL-6 for 3 days and subjected to phagocytosis assays. The results are depicted in Figure 3 . We found that the percentage of phagocytosing cells was reduced in the clones expressing p53 273 (Arg to His) protein, compared to the empty vector, p53-null clone. Thus, the p53 273 (Arg to His) mutant inhibited the differentiation to macrophages to some extent. This inhibitory effect of the p53 273 (Arg to His) mutant is in sharp contrast to the facilitation of differentiation by the p53 143 (Val to Ala) mutant. Therefore, it appears that various p53 mutations may influence differently myeloid differentiation.

\section{Effect of transcription-deficient p53 on myeloid differentiation}

We next investigated the mechanism that underlines the enhancement of myeloid differentiation by the p53 143 (Val to Ala) protein. To examine the possibility that transcription 
activation is required for this process, we utilized transcriptiondeficient p53 forms. ${ }^{39}$ A double mutation changing Leu to GIn at position 22 and Trp to Ser at position 23 (Gln-22, Ser-23) in the p53 transactivation domain (p53 (22-23)) was shown to impair the ability of the wild-type p53 protein to activate transcription. ${ }^{43}$ We examined the effect of the transcriptiondeficient double mutant p53 (22-23), as well as the p53 $22,23,143$ triple mutant, on IL-6-induced myeloid differentiation. First, M1/2 clones expressing the p53 (22-23) were subjected to Giemsa staining. Surprisingly, we revealed that the IL6-mediated differentiation observed in the M1/2 control clone is blocked in M1/2 p53 (22-23)-expressing cells (data not shown). To confirm these findings, we performed a phagocytosis assay for the M1/2 p53 (22-23)-expressing cells, after stimulation with IL-6. As seen in Figure 4, the phagocytic activity in M1/2 cells expressing the p53 (22-23) is markedly reduced, compared to $M 1 / 2$ empty vector cells. Following 3 days with IL-6, $60 \%$ of phagocytosing cells were measured in the empty vector control, while the p53-22, 23-1C cells exhibited $17.5 \%$ of phagocytes and the p53-22, $23-4 \mathrm{C}$ clone did not contain macrophages at all.

Next, we analyzed triple mutant clones expressing the p53 143 (Val to Ala) core mutation in conjunction with the $\mathrm{N}$ terminal 22GIn and 23Ser mutations (p53 22, 23, 143). Cell lines expressing similar levels of either the p53143 (Val to Ala) mutant, the p53 (22-23) double mutant or the p53 22, 23, 143 triple mutant, were compared. ${ }^{39}$ Induction of differentiation was studied both at $32^{\circ} \mathrm{C}$ and at $37^{\circ} \mathrm{C}$. As can be seen in Figure 5 , at $32^{\circ} \mathrm{C}$, wild-type p53 conformation temperature, the enhancement of differentiation by the p53 143 (Val to Ala) was abolished in cells expressing the transcription-defective analog. A similar phenomenon was observed at $37^{\circ} \mathrm{C}$, the temperature for mutant p53 expression. Based on these observations, the transcriptional activity of p53 is necessary to induce p53-dependent cell differentiation, by the p53 143 (Val to Ala) protein. The requirement for p53-mediated transcription is true for both the wild-type and the mutant conformation. Thus, again no difference was observed between the two temperatures. The transcription-deficient p53 (22-23) behaved like the p53 273 (Arg to His) core mutant in the sense that both exerted a blocking effect on differentiation.

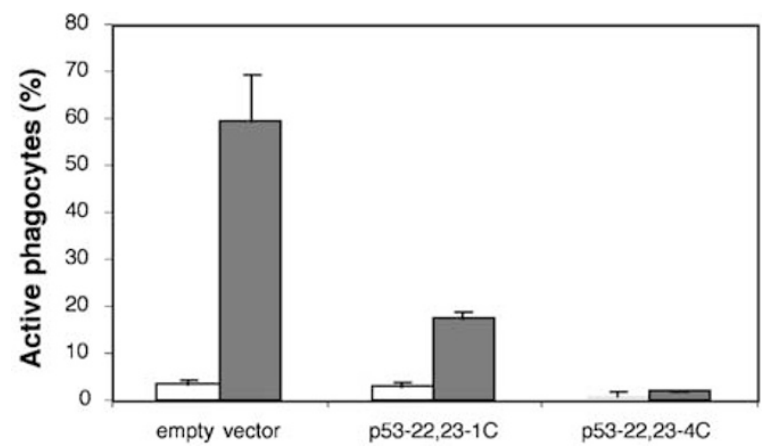

Figure 4 Blockage of myeloid differentiation by p53 (22-23) protein expression. Measurement of phagocytosis of Alexa-labeled E.coli bioparticles by $\mathrm{M} 1 / 2$ cells expressing either empty vector or p53 (22-23). Two clones expressing the p53 (22-23) were utilized: the p53-22, 23-1C and the p53-22, 23$4 \mathrm{C}$. Phagocytosis was assayed following 3 days of incubation with IL-6 (dark bars) or without IL-6 treatment (empty bars)

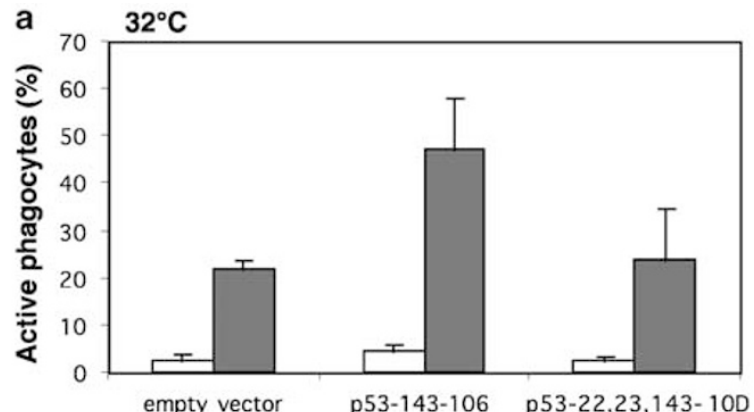

b

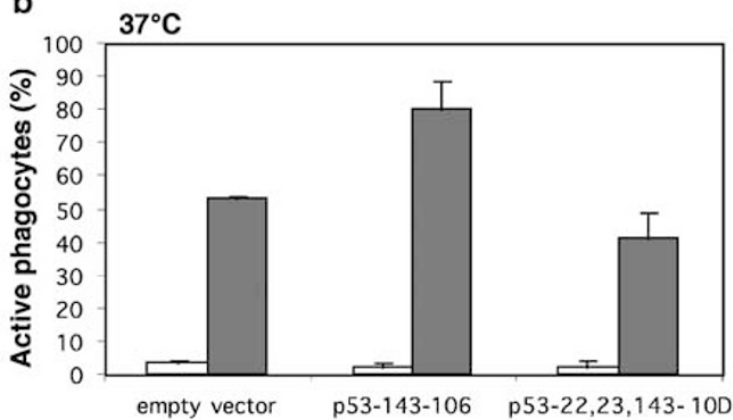

Figure 5 The transcriptional activity of p53 143 (Val to Ala) is essential for its enhancement of differentiation. M1/2 stable clones expressing comparable levels of mutated p53, 143Ala, or 22Gln and 23Ser or these three mutations all together were treated with IL- 6 and shifted to $32^{\circ} \mathrm{C}(\mathbf{a})$, or kept at $37^{\circ} \mathrm{C}(\mathbf{b})$ for 3 days. The phagocytosis of Alexa-labeled E. coli bioparticles, by cells treated with IL-6 (dark bars) or nontreated cells (empty bars), was measured by FACS

\section{Effect of p53 status on the telomerase activity of IL-6-treated M1/2 clones}

To further characterize the differentiation status of $\mathrm{M} 1 / 2$ clones, we measured telomerase activity in the cells, which was shown to be repressed during differentiation. ${ }^{4,45}$ The activity of telomerase was detected by the telomeric repeat amplification protocol (TRAP) assay, before and following 3 days of incubation with IL- 6 at $37^{\circ} \mathrm{C}$ (Figure $6 \mathrm{a}$ ). We found that M1/2 clone containing the p53 143 (Val to Ala) showed enhanced repression of telomerase activity compared to the p53-null control. In contrast, p53 273 (Arg to His)-containing clone did not show any effect. In addition, we found that mutation of the transactivation domain of the p53 143 (Val to Ala) eliminated the enhancement of telomerase repression. These results further confirm our conclusions that p53 143 (Val to Ala) facilitates cell differentiation, while no differentiation is evident with the p53 273 (Arg to His) mutant and with transcription-deficient mutants. To assess in a quantitative manner the kinetics of telomerase activity downregulation, we measured the activity of telomerase using a recently developed real-time TRAP assay. ${ }^{46}$ As shown in Figure $6 \mathrm{~b}$, there was a gradual and mild inhibition of telomerase activity during the course of the experiment in the p53-null control. In contrast, strong downregulation was observed in p53 143 (Val to Ala) containing cells, starting from day 2 of IL- 6 treatment. This lag could be explained by the known long half-life of telomerase.

Next, we performed a kinetic analysis of telomerase activity at $32^{\circ} \mathrm{C}$. The experiment was done in parallel with the one presented in Figure $6 \mathrm{~b}$ to enable direct comparison. As could 
a

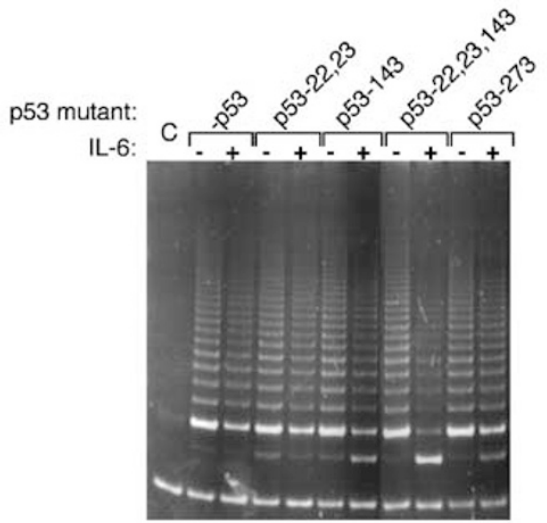

b

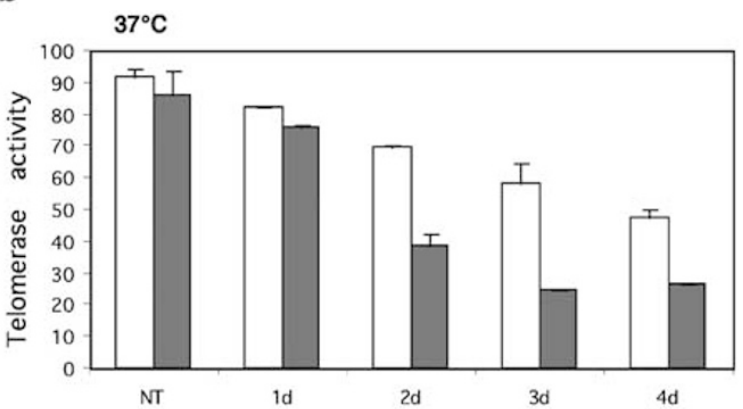

C

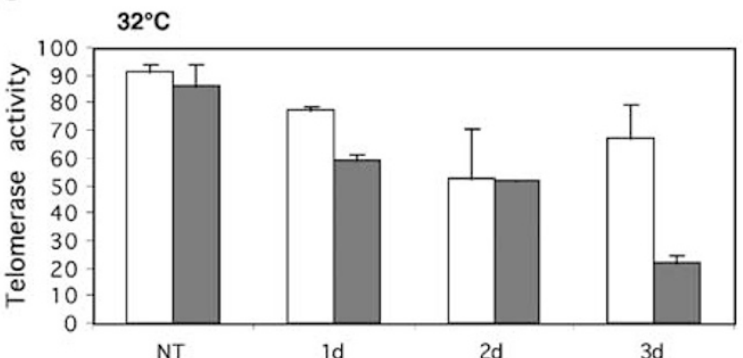

Figure 6 Telomerase activity of M1/2-derived clones before and following IL-6 treatment. Telomerase activity was measured in the following M1/2-derived clones: empty vector clone, p53-22, 23-4C, p53-22, 23,143-10D, p53-143-106 and $\mathrm{p} 53-273-22 \mathrm{~A}$. $\mathrm{C}$, buffer. The intensity of the ladder pattern relative to the internal standard is a measure of telomerase activity (a). Telomerase activity was measured by real-time quantitative PCR. The empty bars represent empty vector clone and the dark bars represent p53-143-106 cells. Cells were treated with IL-6 for the indicated times. The experiment was performed either at $37^{\circ} \mathrm{C}(\mathrm{b})$ or at $32^{\circ} \mathrm{C}(\mathbf{c})$

be seen in Figure $6 c$, there was no significant difference between the p53-null control and p53 143 (Val to Ala) expressing cells up to 2 days of IL- 6 treatment. However, at day 3 , a strong telomerase repression was observed in p53 143 (Val to Ala) containing cells, compared with the p53-null control. These results support our findings of differentiation at $32^{\circ} \mathrm{C}$, using Giemsa staining and phagocytosis assays.

\section{Effect of p53 status on apoptosis induction in IL-6-treated M1/2 clones}

Since we observed an inhibitory effect of the p53 (22-23) protein on IL-6-induced myeloid differentiation, we set experiments to determine the fate of the p53 (22-23) cells

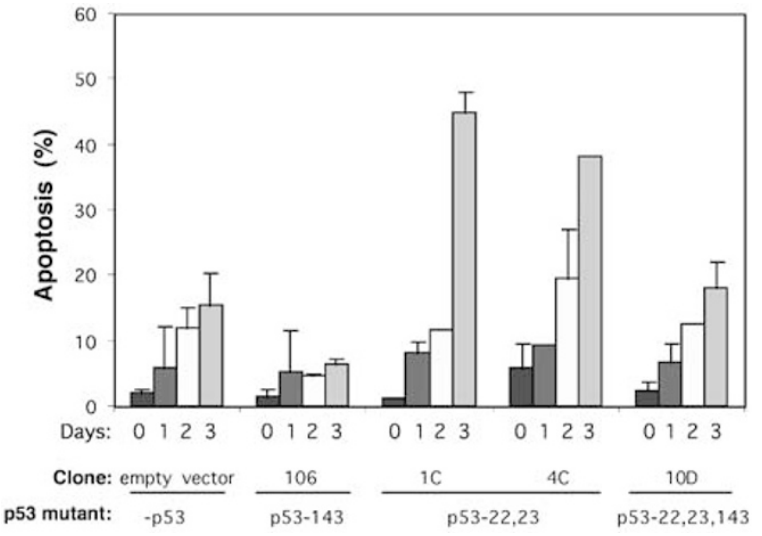

Figure 7 Apoptosis induction in M1/2-derived clones before and following IL-6 treatment. Apoptosis percentage of M1/2 stable clones expressing the p53 143 (Val to Ala), (106), the p53 (22-23), (1C, 4C), the p53-22,23,143, (10D), or the empty vector, following IL-6 treatment at $37^{\circ} \mathrm{C}$ for the time periods indicated. Apoptosis was quantitated by the FACS-based AO DNA denaturability assay

following IL-6 treatment. For that purpose, we analyzed apoptosis of p53 (22-23) expressing M1/2 cells, stimulated with IL-6. M1/2 clones expressing the p53 143 (Val to Ala) and the p53-22,23,143 were included as well. Cell cycle and apoptosis induction were mostly analyzed by the FACS-based AO DNA denaturability assay. ${ }^{42}$ Figure 7 depicts the apoptotic patterns observed before and following IL-6 treatment. Significant differences in the apoptotic percentage of the various clones were mostly observed three days following IL-6 treatment. Consistent with our previous observations, the M1/ 2 clones investigated, including the p53 (22-23)-expressing clone, exhibit a very low percentage of apoptosis in normal growing conditions. ${ }^{39}$ As reported previously for M1 cells, IL6-mediated differentiation was accompanied by a low incidence of apoptosis in $\mathrm{M} 1 / 2$ cells expressing empty vector. Interestingly, the p53-143-106, which expresses the p53 143 (Val to Ala) protein, underwent less apoptosis than the empty vector. Surprisingly, we observed that IL-6 induced a high percentage of apoptotic cells in M1/2 cells expressing the p53 (22-23) transcription-deficient mutant. In addition, the p53 triple mutant-expressing clone exhibited a higher percentage of apoptotic cells compared to the p53 143 (Val to Ala) clone, but significantly less than the p53 (22-23) clones. These findings demonstrate that the expression of the transcriptiondeficient p53 in M1/2 cells not only blocks differentiation, but also induces programmed cell death. Previously, we have observed enhancement of chemotherapy-induced apoptosis by the expression of the p53 (22-23) protein. ${ }^{39}$ Since IL-6 is a physiological agent, the enhancement of apoptosis in the p53 (22-23)-expressing clone following IL-6 induction, further substantiates our conclusions that p53 may facilitate apoptosis in a manner that does not require p53 to be transcriptionally active.

\section{Effect of IL- 6 treatment on p53 protein expression in the M1/2 clones}

Several studies have shown that p53 levels are modulated upon differentiation ${ }^{27-30}$ To determine whether $\mathrm{p} 53$ protein 
a

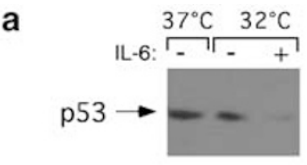

C

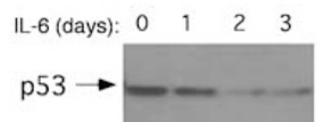

Figure 8 Modulations in p53 protein levels during myeloid differentiation Western blot analysis of p53 143 (Val to Ala) protein expression at $32^{\circ} \mathrm{C}$ after IL-6 treatment for 3 days. The nontreated sample at $32^{\circ} \mathrm{C}$ was kept at this temperature for $6 \mathrm{~h}$. The clone used was the p53-143-106. p53 was detected by the p53 monoclonal antibody pAb1801 (a). Western blot analysis of p53 protein expression in M1/2 clones: p53-143-106, p53-22,23-4C and p53-22,23,143-10D, after 3 days stimulation with IL- 6 at $37^{\circ} \mathrm{C}$. p53 was detected by the p53 monoclonal antibody pAb1801 (b). Kinetics of the reduction in the p53 143 (Val to Ala) protein, using the p53-143-106 clone. Cells were harvested at the indicated time points (days), after treatment with $\mathrm{IL}-6$ at $37^{\circ} \mathrm{C}$, and subjected to Western blot analysis with the p53 polyclonal antibody (c). Quantitation of p53 protein levels in p53-143-103 clone, expressing a low amount of the p53 143 (Val to Ala) protein, after incubation with IL-6 at $37^{\circ} \mathrm{C}$. Cells were harvested at the indicated time points (days) and the p53 protein was detected using the p53 polyclonal antibody (d)

expression is regulated in our experimental system, we have evaluated p53 protein levels in the M1/2-derived clones, before and after IL- 6 treatment. The results are presented in Figure 8. We first determined the effect of IL- 6 stimulation on the expression of the p53 143 (Val to Ala) protein, (clone p53143-106), in the wild-type conformation. Interestingly, we found that pretreatment with IL-6 reduced the expression of the p53 143 (Val to Ala) protein at $32^{\circ} \mathrm{C}$ (Figure 8a). We then examined the effect of IL- 6 exposure at $37^{\circ} \mathrm{C}$, on the expression of several p53 mutants. Western blot analysis with the p53 monoclonal antibody pAb1801 (Figure 8b) revealed that the levels of the p53 (22-23) protein, (clone p53-22, 23-4C), were similar, before and after IL-6 stimulation. Conversely, the p53 143 (Val to Ala) protein levels, (clone p53-143-106), were markedly reduced following IL-6 treatment. This reduction was significantly inhibited in the p53-22, 23, 143-expressing clone, (p53-22, 23, 143-10D), although some reduction was still evident. Moreover, we have observed that the mutant p53 273 (Arg to His) protein levels were slightly reduced following IL-6 induction, but significantly to a lesser extent compared to mutant p53 143 (Val to Ala) protein (data not shown). Taken together, we suggest that a decline in p53 protein levels correlates with involvement of p53 in the myeloid differentiation process.

We next investigated the kinetics of the IL-6-mediated reduction in the p53 143 (Val to Ala) mutant at $37^{\circ} \mathrm{C}$. For that purpose, we used both the p53 monoclonal antibody pAb1801 and the p53 polyclonal antibody. A representative Western blot analysis with the p53 polyclonal antibody is depicted in Figure $8 \mathrm{c}$. We found that, at $48 \mathrm{~h}$ following treatment with IL-6, the p53 143 (Val to Ala) protein levels were reduced. These results were confirmed with the pAb1801 monoclonal antibody. Since a decline in p53 protein levels was demonstrated with both antibodies, we conclude that a true decrease in p53 protein expression occurs, rather than conformational changes.

The decline in p53 143 (Val to Ala) protein was observed utilizing the p53-143-106 clone, which expresses high levels of this protein. The same clone was analyzed in the differentiation and apoptosis assays. We then utilized the p53-143-103 clone, which expresses low levels of the p53 143 (Val to Ala) protein. ${ }^{39}$ As with p53-143-106, the p53-143-103 clone exhibited enhanced differentiation, compared to the empty vector control, both at $37^{\circ} \mathrm{C}$ and $32^{\circ} \mathrm{C}$ (data not shown). Western blot analysis of p53-143-103 cells following IL-6 stimulation revealed that the p53 143 (Val to Ala) protein levels were similar before and after treatment with IL-6 (Figure $8 d$ ). These findings suggest that the participation of p53 in myeloid differentiation requires low levels of p53.

\section{Discussion}

While the involvement of p53 in cell cycle checkpoints and apoptosis is extensively investigated, ${ }^{4,5,47}$ the role of p53 in cellular differentiation is still poorly characterized. Nevertheless, it was shown in several experimental models that wild-type p53 facilitates cellular differentiation (reviewed in Almog and Rotter ${ }^{13}$ ). Indeed, wild-type p53 was found to participate in a variety of differentiation pathways, including muscle cells differentiation, ${ }^{25}$ epithelial cell differentiation, ${ }^{28,48-51}$ B-cell differentiation ${ }^{18-20}$ and erythroid differentiation. ${ }^{23-26}$ Our data support a role for p53 in the myeloid differentiation pathway. We found that the p53 143 (Val to Ala) human temperature-sensitive mutant, at the wild-type conformation, accelerated macrophage differentiation of $\mathrm{M} 1 / 2$ myeloid cells. We observed a differentiation-promoting activity of this protein by analyzing both morphological and functional features of macrophages. These findings are supported by our observations of enhancement of myeloid differentiation in $\mathrm{M} 1 / 2$ clones, which express the murine temperature-sensitive protein, p53 135 (Ala to Val), in the wild-type conformation (data not shown). While previous reports showed the involvement of p53 in myeloid differentiation towards the granulocytic pathway or towards early monocytic differentiation, ${ }^{21,22,65}$ our results show that p53 regulates late monocytic differentiation as well.

Interestingly, the addition of IL- 6 blocked the induction of apoptosis in M1/2 cells expressing p53 143 (Val to Ala) in the wild-type conformation. The results with the $\mathrm{M} 1 / 2$ cells recapitulate similar observations in the parental $M 1$ cell line expressing p53 135 (Ala to Val). ${ }^{41}$ However, in the M1 cell line, the presence of p53, did not affect IL-6-mediated differentiation. On the contrary, we demonstrate that in the M1/2 cells the p53 143 (Val to Ala) protein enhances myeloid differentiation. The apparent discrepancies between the results may be explained by the nature of the $\mathrm{M} 1 / 2$ cells. The $\mathrm{M} 1 / 2$ cell line is dependent for its growth on the presence of growth factors, and thus its differentiation status might be more advanced along the myeloid differentiation pathway than that of the M1 cells.

Surprisingly, in our hands, the human temperature-sensitive p53 143 (Val to Ala) protein, at its mutant conformation, exerted differentiation-promoting effects, as did the protein at 
its wild-type conformation. Likewise, the murine temperaturesensitive mutant, p53 135 (Ala to Val) protein, at the mutant conformation, enhanced the differentiation of M1/2 cells (data not shown). However, the p53 core mutant 273 (Arg to His) inhibited this process. While the p53 143 (Val to Ala) protein is a conformational mutant, the p53 273 (Arg to His) is a DNA contact mutant. Thus, the different impact of these p53 mutants on myeloid differentiation might reflect a difference between the two mutant p53 classes. However, this hypothesis requires further investigation. The notion that mutant p53 might exert a blocking effect on differentiation was demonstrated in several experimental systems, including muscle cells and hematopoietic cells. ${ }^{20,25}$

There are indications that the involvement of p53 in cell differentiation is mediated through its transcriptional activity (reviewed in Almog and Rotter ${ }^{52}$ ). For example, transcriptional activation by p53 was shown to be required for the differentiation of K562 human leukemia cells along the erythroid pathway. ${ }^{25}$ In agreement, we demonstrate in this study that the involvement of p53 in myeloid differentiation is also mediated by a transcription-dependent mechanism. We found, that while the temperature-sensitive p53 143 (Val to Ala), expressed in the wild-type conformation, enhanced macrophage differentiation of $\mathrm{M} 1 / 2$ cells, a transcriptiondefective p53, p53 (22-23), inhibited the differentiation process. Similarly, in $M 1 / 2$ cells expressing the p53 22, 23, 143 protein in the wild-type conformation, the percentage of differentiating cells was significantly reduced. Thus, the participation of wild-type p53 in myeloid differentiation requires a transcriptionally active $\mathrm{p} 53$.

Surprisingly, the transcription-deficient p53 not only lost its capacity to facilitate p53-dependent macrophage differentiation, but also blocked the p53-independent differentiation pathway. These observations suggest that the two pathways are inter-related. Moreover, the interference of the p53 (2223) with the induction of p53-independent differentiation represents a 'gain of function' activity of this protein. In addition to preventing terminal myeloid differentiation, the expression of the p53 (22-23) protein induced apoptosis. Previously, we and others have shown that p53-mediated apoptosis may occur through mechanisms that do not involve transactivation by $p 53 .{ }^{39,53-59}$ We have demonstrated that the expression of the p53 (22-23) protein in M1/2 cells enhances chemotherapy-induced apoptosis. ${ }^{39}$ The observation that the p53 (22-23) mediates apoptosis upon IL-6 stimulation further substantiates our previous results. Moreover, as IL-6 is a physiological agent, our results support the existence of transcription-independent pathway for p53-mediated apoptosis under physiological conditions.

A blockage in myeloid differentiation and induction of apoptosis were also observed upon deregulated expression of the c-myc oncoprotein. ${ }^{60}$ In this case, however, apoptosis was p53-independent. A detailed analysis demonstrated that c-myc induces apoptosis in IL-6-treated myeloid cells through the CD95/Fas pathway. ${ }^{61}$ The Fas pathway was also implicated as a mechanism for p53-mediated apoptosis that is transcription-independent. ${ }^{62}$ Therefore, it would be interesting to examine whether this mechanism underlies the induction of apoptosis by the p53 (22-23) protein, upon exposure to IL-6.
Several studies have shown that endogenous p53 levels are modulated upon differentiation. ${ }^{27-30}$ In support of these findings, we observed a decrease in p53 levels, if the expression level before IL-6 stimuli was high. The p53 143 (Val to Ala) protein, when expressed at high levels in $\mathrm{M} 1 / 2$ cells, was reduced by IL- 6 treatment yet, expression at low levels was unchanged by IL- 6 exposure. These observations suggest that $\mathrm{p} 53$-dependent differentiation may take place when p53 levels are low. It would be of interest to substantiate this hypothesis by further investigation. Interestingly, although the source of the p53 proteins in our experimental system is exogenous, p53 protein expression was regulated during differentiation. This may imply that the differentiation patterns observed with the various p53 forms represent a physiological pathway.

The involvement of p53 in several pathways, including differentiation and apoptosis, raises the ultimate question as to the factors that determine which pathway will be induced following the activation of p53. Although it is still an unresolved issue, several studies pointed to the possibility that p53 levels are an important determinant in selecting the specific response. Induction of p53 expression in both $\mathrm{SaOS}_{2}$ and H1299 p53-null cells induced growth arrest at low p53 levels and apoptosis at high levels of p53. ${ }^{63}$ Likewise, we observed that the levels of genotoxic stress, which reflect the levels of accumulated p53, determine the onset of p53-dependent DNA repair or p53-dependent apoptosis. ${ }^{64}$ In our previous studies, we demonstrated that, while high levels of p53 induce $\mathrm{HL}-60$ cells to undergo apoptosis, low levels of p53 induced, in the same cells, the onset of differentiation. ${ }^{65}$ Here we report on a similar correlation between the levels of p53 and the decision towards differentiation or apoptosis of M1/2 cells. The p53 143 (Val to Ala) protein induces apoptosis when expressed at $32^{\circ} \mathrm{C}$, in the wild-type conformation. However, reduced levels of this protein, by treatment with IL-6, mediate differentiation. It would be interesting to examine whether the reduction in p53 protein expression at $32^{\circ} \mathrm{C}$ is a key event in the blockage of wild-type p53-mediated apoptosis.

In our experimental system, low levels of p53 and p53 transcriptional activation were observed in p53-dependent differentiation. Interestingly, a decrease in p53 protein levels was associated with an increase in transactivation in mouse differentiating keratinocytes ${ }^{28}$ and in retinoic acid-induced differentiation of teratocarcinoma cells. ${ }^{29}$

In recent years, a major effort in generating new drugs for cancer treatment is aimed at the induction of cell differentiation. Particularly, differentiation induction as a treatment for hematologic malignancies has greatly advanced. ${ }^{66} \mathrm{IL}-6$ is one of the cytokines that are currently examined in clinical trials for differentiation therapy. Since we found that different p53 mutants affected differently IL-6-mediated differentiation, it would be interesting to examine the effect of additional p53 mutants on this pathway. Detection of p53 mutants that facilitate IL-6-induced differentiation could offer a powerful approach in the treatment of myeloid cancers, which express these mutants. It should be borne in mind that differentiation induction by the p53 143 (Val to Ala) mutant was coupled with a decrease in its protein levels. Given these observations, it would be important to examine whether other p53 mutant proteins, frequently observed in cancer, are affected similarly 
by IL-6. This would offer a powerful way to modulate the expression of these mutant p53 proteins in cancer cells. Future studies should be aimed at elucidating the molecular mechanisms for these phenomena and examining the clinical implications for cancer therapy.

\section{Materials and Methods}

\section{Cell lines and induction of myeloid differentiation}

The M1/2 cell line was established in our laboratory from the M1, a p53 nonproducer cell line, by the selection for cells that are dependent on medium enriched with growth factors. ${ }^{67}$ Establishment of the M1/2-derived clones expressing various human p53 mutants was described previously. ${ }^{39}$ Clones were continuously grown in DMEM containing $10 \%$ fetal calf serum and conditioned medium. Each set of experiments was performed with the same batch of conditioned medium. Cells were induced for differentiation with $20 \mathrm{ng} / \mathrm{ml}$ human recombinant IL-6 (Calbiochem), after seeding at a concentration of $0.4 \times 10^{5}$ cells $/ \mathrm{ml}$.

\section{Giemsa staining}

Cells were collected at the indicated times following IL- 6 treatment and subjected to cytocentrifugation. The cytospins obtained were fixed with either ethanol or May-Grunwald and then stained with Giemsa to detect morphologic differentiation.

\section{Phagocytosis assays}

Phagocytosis assays were conducted using Alexa-conjugated E. coli bioparticles (Molecular probes) and an $E$. coli opsonising reagent (Molecular probes). Particles were dissolved in PBS containing $0.1 \%$ Azid and sonicated to avoid aggregates. Opsonization was carried out for $1 \mathrm{~h}$ at $37^{\circ} \mathrm{C}$. The particles were then washed three times with PBS. Following harvesting and counting of the cells, cells were incubated with the opsonized particles for $2 \mathrm{~h}$ at $37^{\circ} \mathrm{C}$. A similar set of samples was incubated in ice for $2 \mathrm{~h}$, as a control for absorbence. Cells were then washed three times with PBS and analyzed by the FACSort flow cytometer (Beckton-Dickinson), using the CellQuest (Beckton-Dickinson) software. Acquision of the cells was performed after 1 min of incubation with Trypan blue $(0.04 \%, v / v)$, to quench the signal from particles adsorbed on the cell surface but not yet internalized.

\section{Western blot analysis}

Cell extracts for Western blot analysis were lysed in TLB buffer $(50 \mathrm{mM}$ Tris pH 7.5; $100 \mathrm{mM} \mathrm{NaCl} ; 1 \%$ Triton X-100; 0.5\% Na-deoxycholate; $0.1 \%$ SDS). The samples were normalized according to quantitation by Bradford assay (Bio-Rad). Then, protein sample buffer (140 mM Tris pH 6.8; $22.4 \%$ glycerol; $6 \%$ SDS; $10 \% \beta$-mercaptoethanol and $0.02 \%$ bromophenol blue) was added and the samples were boiled for $10 \mathrm{~min}$ and loaded on $10 \%$ SDS-polyacrylamide gel. The proteins were transferred to nitrocellulose membranes using a semi-dry transfer cell (Bio-Rad). The p53-specific monoclonal antibody PAb-1801 and the p53 polyclonal antibody were used to detect $p 53$. The protein-antibody complexes were detected by using an HRP-conjugated secondary antibody by the super-signal enhanced chemiluminescence system (Pierce). The expression of $\alpha$ tubulin was detected by anti- $\alpha$-tubulin antibody (Sigma), for a quantity control.

\section{FACS analysis of apoptosis}

The AO DNA denaturability assay was performed as previously described. ${ }^{68}$ Briefly, cells were fixed in $80 \%$ ethanol-20\% Hanks balanced salt solution (HBSS) and stored at $-20^{\circ} \mathrm{C}$. Cells were washed once with HBSS, resuspended in HBSS containing $0.25 \mathrm{mg} / \mathrm{ml}$ RNase and incubated at $37^{\circ} \mathrm{C}$ for $1 \mathrm{~h}$ and $15 \mathrm{~min}$

Cells were then harvested and resuspended in HBSS. Cell suspensions $(200 \mu \mathrm{l})$ were added to $0.5 \mathrm{ml}$ of $0.1 \mathrm{M} \mathrm{HCl}$ for $40 \mathrm{~s}$ incubation. Acid denaturation was quenched by the addition of $2 \mathrm{ml}$ of $\mathrm{AO}$ (Molecular Probes) staining solution ( $\mathrm{pH} 2.6$ ), containing $90 \% \mathrm{v} / \mathrm{v} 0.1 \mathrm{M}$ Na-citrate, $10 \% \mathrm{v} / \mathrm{v} 0.2 \mathrm{M} \mathrm{Na}_{2} \mathrm{HPO}_{4}$ and $6 \mu \mathrm{g} / \mathrm{ml} \mathrm{AO}^{42}$

\section{Telomerase activity assays}

Telomerase activity determinations were performed using a commercial TRAPeze kit (Intergene) according to the manufacturer's nonradioactive protocol, except the cycling conditions were modified as follows: $30^{\circ} \mathrm{C}$ $30 \mathrm{~min}, 94^{\circ} \mathrm{C} 3 \mathrm{~min}$, and 29 cycles of amplification $94^{\circ} \mathrm{C}$ for $30 \mathrm{~s}, 56^{\circ} \mathrm{C}$ for $30 \mathrm{~s}, 72^{\circ} \mathrm{C}$ for $30 \mathrm{~s}$. The TRAP reaction was performed using $150 \mathrm{ng}$ lysate.

The real-time quantitative TRAP was performed exactly as described in Wege et al., ${ }^{46}$ using $150 \mathrm{ng}$ of lysate. Relative telomerase activity was calculated from the standard curves generated by serial dilutions of the untreated sample.

\section{Acknowledgements}

This study was supported in part by grants from the Israel-USA Binational Science Foundation (BSF), the Israeli Science Foundation (ISF) and the Kadoori Foundation. VR is the incumbent of the Norman and Helen Asher Professorial Chair in Cancer Research at the Weizmann Institute.

\section{References}

1. Metcalf D (1993) Hematopoietic regulators: redundancy or subtlety? Blood 82 : 3515-3523

2. Metcalf D (1991) Control of granulocytes and macrophages: molecular, cellular, and clinical aspects. Science 254: 529-533

3. Friedman $A D$ (2002) Transcriptional regulation of granulocyte and monocyte development. Oncogene 21: 3377-3390

4. Schwartz D and Rotter V (1998) p53-dependent cell cycle control: response to genotoxic stress. Semin. Cancer Biol. 8: 325-336

5. Sionov RV and Haupt $Y$ (1999) The cellular response to p53: the decision between life and death. Oncogene 18: 6145-6157

6. Ko JL and Prives C (1996) p53: puzzle and paradigm. Genes Dev. 10: 1054-1072

7. El-Deiry WS (1998) Regulation of p53 downstream genes. Semin. Cancer Biol. 8: 345-357

8. Gottlieb TM and Oren M (1998) p53 and apoptosis. Semin. Cancer Biol. 8: 359-368

9. Itahana K, Dimri G and Campisi J (2001) Regulation of cellular senescence by p53. Eur. J. Biochem. 268: 2784-2791

10. Janus F, Albrechtsen N, Dornreiter I, Wiesmuller L, Grosse F and Deppert W (1999) The dual role model for p53 in maintaining genomic integrity. Cell Mol. Life Sci. 55: 12-27

11. Tlsty TD (1997) Genomic instability and its role in neoplasia. Curr Top Microbiol Immunol. 221: 37-46

12. Wahl GM, Linke SP, Paulson TG and Huang LC (1997) Maintaining genetic stability through TP53 mediated checkpoint control. Cancer Surv. 29: 183-219

13. Almog $N$ and Rotter $V$ (1997) Involvement of p53 in cell differentiation and development. Biochim. Biophys. Acta 1333: F1-F27 
14. Donehower LA, Harvey M, Slagle BT, McArthur MJ, Montgomery CA, Butel JS and Bradly A (1992) Mice deficient for p53 are developmentally normal but susceptible to spontaneous tumors. Nature 356: 215-221

15. Sah VP, Attardi LD, Mulligan GJ, Williams BO, Bronson RT and Jacks $T$ (1995) A subset of p53-deficient embryos exhibit exencephaly. Nat. Genet. 10: $175-180$

16. Armstrong JF, Kaufman MH, Harrison DJ and Clarke AR (1995) Highfrequency developmental abnormalities in p53-deficient mice. Curr. Biol. 5: 931-936

17. Choi $\mathrm{J}$ and Donehower LA (1999) p53 in embryonic development: maintaining a fine balance. Cell Mol. Life Sci. 55: 38-47

18. Shaulsky G, Goldfinger N, Peled A, Rotter and V (1991) Involvement of wild type p53 in pre-B cell differentiation in vitro. Proc. Natl. Acad. Sci. USA 88: 8982-8986

19. Shaulsky G, Goldfinger N and Rotter V (1991) Alterations in tumor development in vivo mediated by expression of wild type or mutant p53 proteins. Cancer Res. 51: $5232-5237$

20. Aloni-Grinstein R, Schwartz D and Rotter V (1995) Accumulation of wild-type p53 protein upon gamma-irradiation induces a G2 arrestdependent immunoglobulin kappa light chain gene expression. EMBO J 14: 1392-1401

21. Soddu S, Blandino G, Citro G, Scardigli R, Piaggio G, Ferber A, Calabretta B and Sacchi A (1994) Wild-type p53 gene expression induces granulocytic differentiation of HL-60 cells. Blood 83: 2230-2237

22. Banerjee $D$, Lenz $H$, Schnieders $B$, Manno JD, Ju FJ, Spears $C P$, Hochhauser D, Danenberg K, Danenberg P and Bertino RJ (1995) Transfection of wild-type but not mutant p53 induces early monocytic differentiation in HL60 cells and increases their sensitivity to stress. Cell Growth Differ. 6: 1405-1413

23. Feinstein E, Gale PB, Reed J and Cannai E (1992) Expression of the normal p53 gene induces differentiation of K562 cells. Oncogene 7: 1853-1857

24. Ehinger M, Nilsson E, Persson A, Olsson I and Gullberg U (1995) Involvement of the tumor suppressor gene p53 in tumor necrosis factor-induced differentiation of the leukemic cell line K562. Cell Growth Differ. 6: 9-17

25. Soddu S, Blandino G, Scardigli R, Coen S, Marchetti A, Rizzo MG, Bossi G, Cimino L, Crescenzi M and Sacchi A (1996) Interference with p53 protein inhibits hematopoietic and muscle differentiation. J. Cell Biol. 134: 1-12

26. Johnson P, Chung S and Benchimol S (1993) Growth suppression of Friend virus-transformed erythroleukemia cells by p53 protein is accompanied by hemoglobulin production and is sensitive to erythropoitin. Mol. Cell. Biol. 13: 1456-1463

27. Kastan MB, Radin Al, Kuerbitz SJ, Onyekwere O, Wolkow CA, Civin Cl, Stone KD, Woo T, Ravindranath Y and Craig RW (1991) Levels of p53 protein increase with maturation in human hematopoietic cells. Cancer Res. 51: 4279-4286

28. Weinberg CW, Azzoli GC, Chapman K, Levine JA and Yuspa HS (1995) p53mediated transcriptional activity increases in differentiating epidermal keratinocytes in association with decreased p53 protein. Oncogene 10: 2271-2279

29. Lutzker SG and Levine AJ (1996) A functionally inactive p53 protein in teratocacinoma cells is activated by either DNA damage or cellular differentiation. Nat. Med. 2: 804-810

30. Sabapathy K, Klemm M, Jaenisch R and Wagner EF (1997) Regulation of ES cell differentiation by functional and conformational modulation of $\mathrm{p} 53$. EMBO J. 16: 6217-6229

31. Aloni-Grinstein R, Zan-Bar I, Alboum I, Goldfinger N and Rotter V (1993) Wild type p53 functions as a control protein in the differentiation pathway of the Bcell lineage. Oncogene 8: 3297-3305

32. Weintraub H, Hauschka S and Tapscott SJ (1991) The MCK enhancer contains a p53 responsive elements. Proc. Natl. Acad. Sci. USA 88: 4570-4571

33. Saifudeen Z, Dipp S and El-Dahr SS (2002) A role for p53 in terminal epithelial cell differentiation. J. Clin. Invest. 109: 1021-1030

34. Hussain SP and Harris CC (1998) Molecular epidemiology of human cancer: contribution of mutation spectra studies of tumor suppressor genes. Cancer Res. 58: 4023-4037

35. Feinstein E, Cimino G, Gale RP, Alimena G, Berthier R, Kishi K, Goldman J, Zaccaria A, Berrebi A and E C (1991) p53 in chronic myelogenous leukemia in acute phase. Proc. Natl. Acad. Sci. USA 88: 6293-6297
36. Fagin JA (1995) Tumor supresor genes in human thyroid neoplasma: p53 mutations are associated with undifferentiated thyroid cancers. J. Endocrinol. Invest. 18: 140-142

37. Miyaura C, Onozaki K, Akiyama $Y$, Taniyama T, Hirano T, Kishimoto $T$ and Suda T (1988) Recombinant human interleukin 6 (B-cell stimulatory factor 2) is a potent inducer of differentiation of mouse myeloid leukemia cells (M1). FEBS Lett. 234: 17-21

38. Peled A, Lee BC, Toledo J, Aracil M and Zipori D (1996) Interactions between leukemia cells and bone marrow stromal cells: stroma-supported growth vs. serum dependence and the roles of TGF- $\beta$ and M-CSF. Exp. Hematol. 24: 728-737

39. Matas D, Sigal A, Stambolsky P, Milyavsky M, Weisz L, Schwartz D, Goldfinger $\mathrm{N}$ and Rotter V (2001) Integrity of the N-terminal transcription domain of p53 is required for mutant $\mathrm{p} 53$ interference with drug-induced apoptosis. EMBO J. 20: 4163-4172

40. Zhang W, Guo XY, Hu GY, Liu WB, Shay JW and Deisseroth AB (1994) A temperature-sensitive mutant of human p53. EMBO J. 13: 2535-2544

41. Yonish-Rouach E, Resnitzky K, Lotem J, Sachs L, Kimchi A and Oren M (1991) Wild type p53 induces apoptosis of myeloid leukemic cells that is inhibited by interleukin 6. Nature 352: 345-347

42. Darzynkiewicz Z, Li X and Gong J (1994) Assays of cell viability: Discrimination of cell dying by apoptosis. Methods Cell Biol. 41: 15-38

43. Lin J, Chen J, Elenbaas B and Levine AJ (1994) Several hydrophobic amino acids in the p53 amino-terminal domain are required for transcriptional activation, binding to mdm-2 and the adenovirus 5 E1B $55-k D$ protein. Genes Dev. 8: 1235-1246

44. Nozawa K, Maehara K and Isobe K (2001) Mechanism for the reduction of telomerase expression during muscle cell differentiation. J. Biol. Chem. 276: 22016-22023

45. Mattson MP and Klapper W (2001) Emerging roles for telomerase in neuronal development and apoptosis. J. Neurosci. Res. 63: 1-9

46. Wege H, Chui MS, Le HT, Tran JM and Zern MA (2003) SYBR Green real-time telomeric repeat amplification protocol for the rapid quantification of telomerase activity. Nucleic Acids Res. 31: E3-3

47. Vousden KH (2000) p53: death star. Cell 103: 691-694

48. Brenner L, Teresita M-A, Vellucci VF, Zhou Z-L and Reiss M (1993) Wild-type p53 tumor suppressor gene restores differentiation of human squamous carcinoma cells but not the response to transforming growth factor $\beta^{1}$. Cell Growth Differ. 4: 993-1004

49. Woodworth CD, Wang H, Simpson S, Alvarez-Salas LM and Notario V (1993) Overexpression of wild-type p53 alters growth and differentiation of normal human keratinocytes but not human papillomavirus-expressing cell lines. Cell Growth Differ. 4: 367-376

50. Spandau DF (1994) Distinct conformations of p53 are observed in different stages of keratinocyte differentiation. Oncogene 9: 1861-1868

51. Rehberger PA, Richter KH, Schwartz D, Goldfinger N, Oskato R, Almog N, Marks F and Rotter V (1997) Differential expression of the regularly spliced wild-type p53 and its $\mathrm{COOH}$-terminal alternatively spliced form during epidermal differentiation. Cell Growth Differ. 8: 851-860

52. Almog $N$ and Rotter $V$ (1997) Involvement of $p 53$ in cell differentiation and developmemt. Biochim. Biopyhs. Acta 1333: F1-F27

53. Caelles C, Helmberg A and Karin M (1994) p53-dependent apoptosis in the absence of transcriptional activation of p53-target genes. Nature 370 : 220-223

54. Wagner AJ, Kokontis JM and Hay N (1994) Myc-mediated apoptosis requires wild-type p53 in a manner independent of cell cycle arrest and the ability of p53 to induce p21(waf1/cip1). Gene Dev. 8: 2817-2830

55. Haupt Y, Rowan S, Shaulian E, Vousden KH and Oren M (1995) Induction of apoptosis in HeLa cells by trans-activation-deficient p53. Genes Dev. 9: 2170-2183

56. Haupt Y, Rowan S, Shaulian E, Kazaz A, Vousden K, Oren and M (1997) p53 mediated apoptosis in HeLa cells: transcription dependent and independent mechanisms. Leukemia 11 (Suppl 3): 337-339

57. Yan Y, Shay JW, Wright WE and Mumby MC (1997) Inhibition of protein phosphatase activity induces p53-dependent apoptosis in the absence of p53 transactivation. J Biol Chem 272: 15220-15226

58. Ding HF, Lin YL, McGill G, Juo P, Zhu H, Blenis J, Yuan J and Fisher DE (2000) Essential role for caspase-8 in transcription-independent apoptosis triggered by p53 (in process citation). J. Biol. Chem. 275: 38905-38911 
59. Kokontis JM, Wagner AJ, O'Leary M, Liao S and Hay N (2001) A transcriptiona activation function of p53 is dispensable for and inhibitory of its apoptotic function. Oncogene 20: 659-668

60. Amanullah A, Liebermann DA and Hoffman B (2000) p53-independent apoptosis associated with c-Myc-mediated block in myeloid cell differentiation. Oncogene 19: 2967-2977

61. Amanullah A, Liebermann DA and Hoffman B (2002) Deregulated c-Myc prematurely recruits both Type I and II CD95/Fas apoptotic pathways associated with terminal myeloid differentiation. Oncogene 21: 1600-1610

62. Bennet M, Macdonald K, Chan S-W, Luzio JP, Simari R and Weissberg P (1998) Cell surface trafficking of fax: a rapid mechanism of p53-mediated apoptosis. Science 282: 290-293

63. Chen X, Ko LJ, Jayaraman L and Prives C (1996) p53 levels, functional domains, and DNA damage determine the extent of the apoptotic response of tumor cells. Genes Dev 10: 2438-2451
64. Offer H, Erez N, Zurer I, Tang X, Milyavsky M, Goldfinger N and Rotter V (2002) The onset of p53-dependent DNA repair or apoptosis is determined by the level of accumulated damaged DNA. Carcinogenesis 23: 1025-1032

65. Ronen D, Schwartz D, Teitz Y, Goldfinger N and Rotter V (1996) Induction of $\mathrm{HL}-60$ cells to undergo apoptosis is determined by high levels of wild-type p53 protein whereas differentiation of the cells is mediated by lower p53 levels. Cell Growth Differ 7: 21-30

66. Miller Jr WH and Waxman S (2002) Differentiation induction as a treatment for hematologic malignancies. Oncogene 21: 3496-3506

67. Peled A, Zipori D and Rotter V (1996) Cooperation between p53-dependent and p53-independent apoptotic pathways in myeloid cells. Cancer Res 56: 2148-2156

68. Li R, Sutphin PD, Schwartz D, Matas D, Almog N, Wolkowicz R, Goldfinger N, Pei H, Prokocimer M and Rotter V (1998) Mutant p53 protein expression interferes with p53-independent apoptotic pathways. Oncogene 16: 3269-3277 